\title{
COHERENCE FOR CATEGORIES WITH ASSOCIATIVITY, COMMUTATIVITY AND DISTRIBUTIVITY
}

\author{
BY MIGUEL L. LAPLAZA
}

Communicated by Saunders Mac Lane, August 5, 1971

Let $\mathscr{C}$ be a category, $\otimes, \oplus: \mathscr{C} \times \mathscr{C} \rightarrow \mathscr{C}$ two functors, $U$ and $N$ objects of $\mathscr{C}$. Suppose that for any objects, $A, B, C$ of $\mathscr{C}$ we have natural isomorphisms,

$$
\begin{aligned}
\alpha_{A, B, C}: A \otimes(B \otimes C) \rightarrow & (A \otimes B) \otimes C, \\
\alpha_{A, B, C}^{\prime}: A \oplus(B \oplus C) \rightarrow & (A \oplus B) \oplus C, \\
\lambda_{A}: U \otimes A \rightarrow A, \quad & \lambda_{A}^{\prime}: N \oplus A \rightarrow A, \\
\rho_{A}: A \otimes U \rightarrow A, \quad & \rho_{A}^{\prime}: A \oplus N \rightarrow A, \\
\gamma_{A, B}: A \otimes B \rightarrow B \otimes A, \quad & \gamma_{A, B}^{\prime}: A \oplus B \rightarrow B \oplus A, \\
& \lambda_{A}^{*}: N \otimes A \rightarrow N, \\
& \rho_{A}^{*}: A \otimes N \rightarrow N,
\end{aligned}
$$

and natural monomorphisms

$$
\begin{aligned}
& \delta_{A, B, C}: A \otimes(B \oplus C) \rightarrow A \otimes B \oplus A \otimes C, \\
& \delta_{A, B, C}^{\prime}:(A \oplus B) \otimes C \rightarrow A \otimes C \oplus B \otimes C .
\end{aligned}
$$

Roughly speaking the coherence problem is to determine the conditions (denoted coherence conditions) in which the arrows obtained by combining elements of type (I), (II) and identities with $\otimes$ and $\oplus$ only depend on the domain and codomain of the arrow. This note is to announce an answer to this question that was proposed in [6] as raised by $\mathrm{H}$. Bass.

The first coherence results stated as such are contained in [5] which treats the case of only one functor $\otimes$. The solution for a more complicated situation in closed categories is given in [4]. Other papers with results related to coherence problems are listed in the references.

Take a set, $X=\left\{x_{1}, x_{2}, \ldots, x_{p}, n, u\right\}$, and construct $\mathscr{A}$, the free $\{\cdot,+\}$-algebra over it. Let $\mathscr{G}$ be the graph of all formal symbols, for $x, y, z \in \mathscr{A}$,

AMS 1970 subject classifications. Primary 18D99; Secondary 18D10.

Key words and phrases. Coherent, coherence conditions, distributivity, regular element, graph. 


$$
\begin{gathered}
\alpha_{x, y, z}: x(y z) \rightarrow(x y) z, \quad \alpha_{x, y, z}^{\prime}: x+(y+z) \rightarrow(x+y)+z \\
\lambda_{x}: u x \rightarrow x, \quad \lambda_{x}^{\prime}: n+x \rightarrow x, \\
\rho_{x}: x u \rightarrow x, \quad \rho_{x}^{\prime}: x+n \rightarrow x, \\
\gamma_{x, y}: x y \rightarrow y x, \quad \gamma_{x, y}^{\prime}: x+y \rightarrow y+x, \\
\lambda_{x}^{*}: n x \rightarrow n, \\
\rho_{x}^{*}: x n \rightarrow n,
\end{gathered}
$$

their formal inverses and

$$
\begin{aligned}
\delta_{x, y, z}: x(y+z) & \rightarrow x y+x z, \\
\delta_{x, y, z}^{\prime}:(x+y) z & \rightarrow x z+y z, \\
1_{x}: x & \rightarrow x .
\end{aligned}
$$

Construct $\mathscr{H}$, the free $\{\cdot,+\}$-algebra over $\mathscr{G}$ and take on $\mathscr{H}$ the only extension of the graph structure of $\mathscr{G}$ in which the projections are $\{\cdot,+\}$ morphisms. One element of $\mathscr{H}$ is said to be an instantiation if, with at most one exception, only elements of $\mathscr{G}$ of type $1_{x}$ occur in its expression. We will denote by $\mathscr{I}$ the graph of all the instantiations of $\mathscr{G}$.

Fix now $p$ objects, $C_{1}, C_{2}, \ldots, C_{p}$, of $\mathscr{C}$ and let $g: \mathscr{I} \rightarrow \mathscr{C}$ be the morphism of graphs defined on the vertices by the conditions (i) $g u=U$, $g n=N, g x_{i}=C_{i}$, for $1 \leqq i \leqq p$, (ii) $g(x+y)=g x \oplus g y, g(x y)=g x \otimes g y$, for $x, y \in \mathscr{A}$, on $\mathscr{G}$ by taking each formal symbol onto the arrow of $\mathscr{C}$ determined replacing each subscript by its image by $g$ and such that for $x, y \in \mathscr{I}, g(x+y)=g x \oplus g y, g(x y)=g x \otimes g y$. This definition depends upon the $C_{i}$ and allows us to define the value of a path with steps in $\mathscr{I}$ as the product of the images of the steps.

Let $\mathscr{A}^{*}$ be the free $\{\cdot,+\}$-algebra over $X$, with associativity and commutativity for - and + , distributivity of - relatively to + , null element $n$, identity element $u$, and the additional condition $n a=n$ for $a \in \mathscr{A}^{*}$. The identity map of $X$ defines a $\{\cdot,+\}$-morphism $f: \mathscr{A} \rightarrow \mathscr{A}^{*}$. An element, $a$, of $\mathscr{A}$ is defined to be regular if $f a$ can be expressed as a sum of different elements of $\mathscr{A}^{*}$, each of which is a product of different elements of $X$.

Our coherence conditions require the commutativity of the diagrams of a finite family of types. Roughly speaking our conditions are equivalent to the commutativity of any diagram that can be constructed taking, for each vertex, the iteration by $\otimes$ and $\oplus$ of not more than four objects, equal or different, of $\mathscr{C}$ and such that each edge is an iteration by $\otimes$ and $\oplus$ of arrows of type (I), (II) and identities: we are reduced to a finite number of types of diagrams if we drop unnecessary commutativity conditions. 
With the above definitions we can state the following theorem which is our main result.

COHERENCE THEOREM. If $\mathscr{C}$ satisfies the coherence conditions and $a$ is regular then the value of any path from a to $b$, whose steps are in $\mathscr{I}$, depends only upon $a$ and $b$.

A detailed exposition of these results will appear elsewhere.

\section{REFERENCES}

1. J. Bénabou, Structures algébriques dans les catégories, Cahiers Topologie Géom. Différentielle, 10 (1968), 1-126. MR 39 \# 5650.

2. D. B. A. Epstein, Functors between tensored categories, Invent. Math. 1 (1966), 221-228. MR 35 \# 4276.

3. G. M. Kelly, On Mac Lane's conditions for coherence of natural associativities, commutativities, etc., J. Algebra 1 (1964), 397-402. MR 32 \# 132.

4. G. M. Kelly and S. Mac Lane, Coherence in closed categories, J. Pure Appl. Algebra 1 (1971), 97-140.

5. S. Mac Lane, Natural associativity and commutativity, Rice Univ. Studies 49 (1963), no. 4, 28-46. MR 30 \# 1160.

6. Coherence and canonical maps, Sympos. Mathematica 4 (1970), 231-241.

Department of Mathematics, University of Chicago, Chicago, Illinois 60637 00708

Department of Mathematics, University of Puerto Rico, Mayaguez, Puerto Rico 